\title{
Single-Image Calibration of Off-Axis Catadioptric Cameras Using Lines
}

\author{
Vincenzo Caglioti, Pierluigi Taddei, Giacomo Boracchi, Simone Gasparini, Alessandro Giusti \\ Politecnico di Milano \\ Dipartimento di Elettronica e Informazione \\ Piazza Leonardo da Vinci, 32 \\ I-20133 Milano (MI), Italy \\ \{name.surname\}apolimi.it
}

\begin{abstract}
We present a novel calibration method for off-axis catadioptric cameras, i.e. standard perspective cameras placed in a generic position w.r.t. an axial-symmetric mirror of unknown shape. The proposed method estimates the intrinsic parameters of the natural perspective camera, the $3 D$ shape of the mirror and its pose w.r.t. the camera.

The peculiarity of our approach is that, unlike several other calibration methods, we do not require any cross section of the mirror to be visible in the image. Instead, we require that the catadioptric image contains at least the image of one generic space line. We then derive some constraints that, combined with the harmonic homology relating the apparent contours of the mirror, allow us to calibrate the off-axis camera. We provide experimental results both on synthetic and camera images that prove the validity of the technique.
\end{abstract}

\section{Introduction}

We present a novel method for calibrating non-central catadioptric cameras with an axial-symmetric mirror whose profile $^{1}$ is unknown. In catadioptric cameras the viewing rays coming from the scene are reflected by the mirror before they pass through the camera viewpoint and they cross the image plane. A catadioptric camera is said to be calibrated if the viewing ray associated to each image point is known.

Catadioptric cameras where all the viewing rays are concurrent to the same point are said to be central, as stated by Baker and Nayar [2]. Their mirror is a quadric of revolution, with the viewpoint of the perspective camera placed at one of its foci.

In noncentral cameras viewing rays are not all concurrent at a common point. In this paper we consider non-

\footnotetext{
${ }^{1}$ By profile, we mean the SOR generator
}

central catadioptric cameras, whose mirror is a surface of revolution (SOR) of unknown profile (axial symmetric mirrors are easy to manufacture). In particular, we consider off-axis catadioptric cameras, i.e. cameras whose viewpoint is placed at a generic position out of the mirror axis. Unlike central cameras, noncentral cameras allow one to localize space lines [3,14] or spheres [4] from a single image. This peculiarity makes them particularly suited for the 3D reconstruction of indoor scenes: in fact, the single-image approach, unlike methods based on multiple views, does not require the solution of the correspondence problem, and, unlike laser-based methods, is not invasive. Obviously, for $3 \mathrm{D}$ reconstruction, a calibrated system is needed.

Several calibration methods exist for central catadioptic cameras, most of which depend on the mirror shape and exploit images of lines [10,21], spheres [20] or calibration patterns [13]. Rather few methods have been proposed for calibrating noncentral cameras; the first attempt was made by Aliaga [1] who introduced a paracatadioptric camera calibration method that relaxes the assumption of the perfect orthographic projection and placement. Later, Mashita et al. in [15] presented a calibration method for noncentral catadioptric cameras based on a SOR mirror. In particular, they devised a localization procedure for an off-axis catadioptric camera based on an hyperboloidal mirror [19]. Their method estimates the extrinsic parameters of the camera, i.e. the mirror pose with respect to the camera, using the imaged ellipse corresponding to a circular cross section of the mirror; they exploit the calibration method devised for standard perspective cameras by Chen et al. [7] and Sun et al. [17]. Both these methods are based on the algorithm described by Kanatani in [12], which recovers the 3D orientation of a circle in the scene from its projected image. Sun's method estimates the intrinsic parameters of a standard perspective camera using images with three or more concentric conics under different views. Chen's method estimates the focal length and the extrinsic parameters using a single image with at least two coplanar circumferences. Moreover, 
Fabrizio et al. in [9] use the mirror boundaries as a calibration pattern for estimating the mirror pose by computing the homographies among the image plane and the planes containing the two boundaries. It is worth noting that all cited methods assume that the mirror profile is exactly known, which requires high manufacturing accuracy.

We propose a calibration method for off-axis cameras, whose mirror has unknown profile. Off axis cameras are not dependent on exact alignment of the camera and mirror, which is often a problem in axial systems. We require a single image, depicting the mirror apparent contours and the catadioptric image of at least one space line in a generic, unknown position. Our method does not require any circular cross section of the mirror to be visible. The image of the mirror axis is obtained by computing the harmonic homology that relates the mirror apparent contours [8]. Then we exploit additional constraints deriving from the image of a space line: in fact, the viewing rays associated to these image points have to intersect a single space line. Thus, we recover the intrinsic camera parameters, the camera position w.r.t. the mirror axis and the mirror profile.

After formalizing the problem in Section 2, we detail the proposed calibration method and its implementation in Sections 3, 4 and 5. In Section 6 we present some experimental results validating the method, while Section 7 concludes and presents ongoing works.

\section{Problem formulation}

We consider an off-axis catadioptric camera, based on a natural camera (null skew factor and unit apsect ratio $^{2}$ ). We assume that the mirror's lateral apparent contour is visible in the image, without requiring that any circular cross section (such as the mirror base, or the mirror top) is visible. Moreover, the catadioptric image of at least a space line must be available; we also assume that this line satisfies the broad conditions in [3].

Our goal is to calibrate the catadioptric system, estimating the intrinsic camera parameters and, up to a scale factor, the mirror profile and the mirror position w.r.t. the camera viewpoint.

In the following section we briefly introduce the harmonic homology and the notation used in the rest of the paper.

\section{Harmonic Homology and Off-axis Cameras}

Let $\pi$ be the symmetry plane containing the mirror axis $L$ and the camera viewpoint $O$ (see Figure 1). Let also $\gamma$ be the mirror apparent contour. The relation between the points on the two facing parts of $\gamma$ is described by the harmonic

\footnotetext{
${ }^{2}$ nonunit, but known, aspect ratio can easily be reduced to unit aspect
}

homology $H$ :

$$
H=I-2 \frac{v \cdot l^{T}}{v^{T} \cdot l}
$$

being $I$ the $3 \times 3$ identity matrix, $l$ the image of the mirror axis, and $v$ the vanishing point of the direction orthogonal to $\pi$.

Recently, Colombo et al. in [8] devised a method for computing the harmonic homology given a single perspective image of a SOR. They use the harmonic homology and the image of at least a circular cross section for calibrating a perspective camera. In our work, we use their technique for computing the harmonic homology, and, thus, $v$ and $l$, which are used as a constraint for camera calibration. On the other hand, we do not assume that a cross section is visible, as detailed in the following section.

\section{Technique}

\subsection{Reduction to two parameters}

According to the natural camera assumption, the only intrinsic parameters to be estimated are the principal point and the focal length. Moreover, we exploit $l$ and $v$, which are derived from $\gamma$ as previously described. Consider now a generic point $p$ on $l$ : since its viewing ray is orthogonal to the direction represented by vanishing point $v$, the viewpoint of the perspective camera is constrained to belong to the sphere whose diameter is $v p$. In particular, let $\bar{p}$ be the orthogonal projection of $v$ on $l$ : the camera viewpoint $O$ is constrained to belong to the circumference with diameter $v \bar{p}$, lying on a plane perpendicular to the image plane.

Let $\alpha$ be the angle between the mirror axis $L$ and its projection $l$. If the perspective camera is calibrated and $\alpha$ is known, the mirror profile and pose can be determined (see Figure 1). This holds up to a scale factor which we will disregard in what follows; this scale factor can be fixed if any length in the scene (such as the maximum mirror radius) is known.

Once the mirror profile, the camera pose and the camera calibration matrix have been estimated, it is possible to determine the viewing ray associated to any image point. Therefore, in order to calibrate the catadioptric camera, one has to estimate the parameter $k$ (which uniquely defines the perspective camera intrinsic calibration matrix), as well as the angle $\alpha$. This is accomplished by exploiting the image of a space line, as explained in the following paragraph.

\subsection{Exploiting the image of a space line}

Two image points $p$ and $q$ on $\gamma$ are said to be corresponding if they are the images of two points on the mirror surface which are symmetric with respect to the plane $\pi$. In other words, $p$ and $q$ are the projection of points lying on the same cross section $C$ of the mirror. The harmonic homology $H$ allows us to identify corresponding points on $\gamma$. 


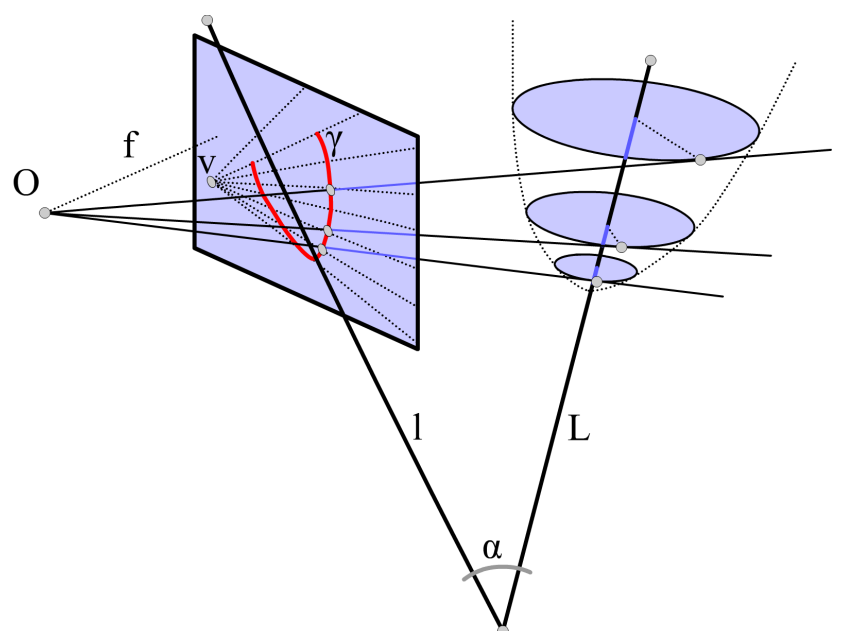

Figure 1. The apparent contour on the image plane and a subset of cross sections related to some point of $\gamma$ are shown.

Let $p$ and $q$ be a pair of corresponding points, and let $c$ be the image of $C: c$ is a conic, tangent to $\gamma$ in $p$ and $q$. Since the tangents to $\gamma$ are easily computed, and five constraints determine a planar conic, only one residual parameter defines $c$. This parameter can be expressed as a function of the two unknowns $k$ and $\alpha$. In addition, the normal to the mirror surface at any point of $C$ is also known if $k$ and $\alpha$ are given.

Consider six (or more) pairs $\left(p_{i}, q_{i}\right)$ of corresponding points on $\gamma$, and consider the image of a space line, consisting in a curve $s$. Let $c_{i}$ be the imaged cross sections through $\left(p_{i}, q_{i}\right)$, and let $b_{i}$ be the intersection between $s$ and $c_{i}$ : among the named entities, $c_{i}, b_{i}$ and the associated viewing rays can be expressed as functions of the two unknowns. Note that the relationship between the two unknowns and the viewing rays can not be expressed analytically, because $s$ has no analytical form. However, an approximate expression of the partial derivatives of the viewing rays parameters with respect to the unknowns can be obtained; these are then exploited in the subsequent nonlinear minimization (see Section 5).

Consider four of the six viewing rays: there are two possible space lines crossing all the four viewing rays [11]. These two lines can be also expressed in terms of the two unknown parameters. Two equations in the two unknowns $\alpha$ and $k$ can then be obtained by constraining the two residual viewing rays to cross the same space line. These are sufficient to determine the unknown parameters.

However, for increased robustness, we can use additional constraints which can be obtained by either considering more than six viewing rays from a single image curve, or taking into account multiple images of space lines, when available.

Once the two unknown parameters have been determined, the mirror axis orientation $\alpha$ is known, together with the profile of the part of the mirror whose apparent contours are visible in the image.

Any other cross section whose image does not cross the apparent contour $\gamma$ is still unknown. These can be reconstructed, provided that they cross the image of at least two lines whose 3D position has already been determined.

\section{Implementation notes}

We implemented the calibration method as a nonlinear minimization of an error term $e$, which we define in the following. For any parameters pair $(\alpha, k)$, we compute the position in space of the mirror axis $L$, and we recover the mirror profile from $\gamma$. Then we consider the image $s$ of a space line and we compute the viewing rays associated to several points of $s$. These viewing rays constitute a viewing surface.

If we consider a single line, the error term $e(\alpha, k)$ expresses how far the viewing surface is from containing a generic space line; we consider $e(\alpha, k)$ to be the reconstruction error residual $r(\alpha, k)$ : it is computed using a robust method for line localization given a set of viewing rays. The method is derived from [3] and it is explained in details in [5].

In order to improve robustness, more than one image line can be exploited: the error term $e(\alpha, k)$ becomes a weighted average of the reconstruction error residuals $r_{i=1 . . n}$. The weight for each viewing surface is given by a nonnegative quadric similarity measure $m_{i=1 . . n}(\alpha, k)$, which expresses how far the viewing surface is from being a ruled quadric. If the viewing surface is a ruled quadric $(m(\alpha, k)=0)$, it contains infinite lines besides the viewing rays, therefore the associated residual is not reliable. Conversely, high values of $m(\alpha, k)$ correspond to residuals better related to the quality of the calibration parameters.

In particular, given a set of $n$ image curves $s_{i=1 . . n}$, the error term $e(\alpha, k)$ is given by

$$
e(\alpha, k)=\frac{\sum_{i}^{n} r_{i}(\alpha, k) / m_{i}(\alpha, k)}{\sum_{i}^{n} 1 / m_{i}(\alpha, k)} .
$$

We refer the reader to [5] for more details on how to calculate the quadric similarity given a set of straight line lying on a ruled surface.

In order to speed up the process and improve robustness to outliers, it is possible to initially compute the quadric similarity for all surfaces, and evaluating the reconstruction residual only for the subset of surfaces with highest reconstruction quality.

Our implementation of the technique is currently using a stratified approach, in which the camera focal length is known a-priori. The principal point is thus directly determined using the harmonic homology $H$. In this case, the problem becomes a bounded minimization of the residual error $e$ on the single parameter $\alpha$. In case $k$ is also consid- 


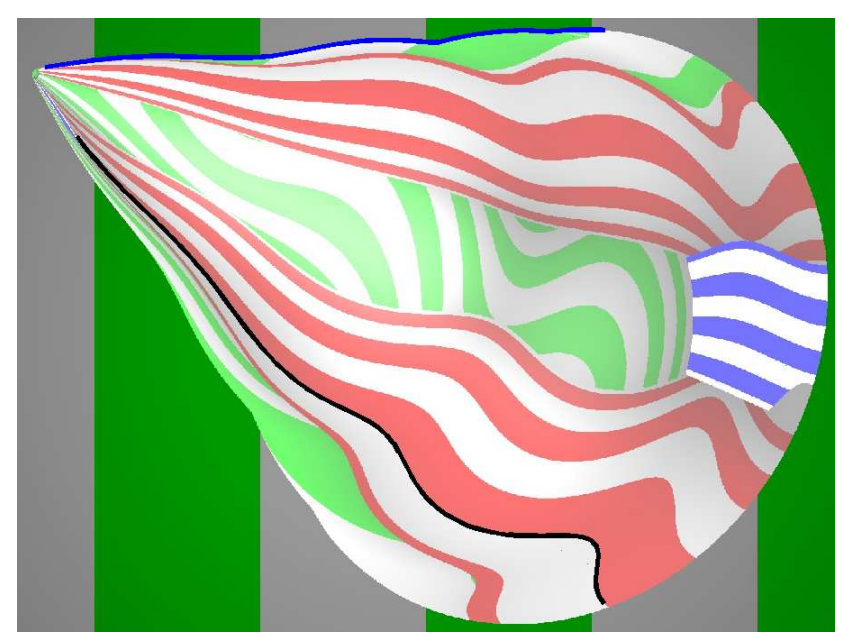

Figure 2. A raytraced mirror; one of the two apparent contours is represented in blue whereas the line image used for the minimization is shown in black. Note a visible cross section (the mirror base), which is not exploited by our technique.

ered, we would have to minimize the residual error on $k$ and $\alpha$ simultaneously.

\section{Experimental results}

The proposed calibration method has been tested both on synthetic and camera images.

Synthetic images have been generated using the raytracer software POV-Ray [16]; examples of said images are represented in Figure 2 and Figure 4. These experiments have been designed for validating the calibration method. Therefore we assumed that the mirror axis projection $l$, the vanishing point $v$, and the intrinsic camera parameters were known a priori. The mirror apparent contour $\gamma$ and some images of scene lines have been extracted using the canny edge detector [6]. The error term for the considered $\alpha$ values (angles between $-90^{\circ}$ and $90^{\circ}$ at $1 / 6^{\circ}$ step) has been computed with (2). The estimated $\alpha$ value is the one having the minimum error term.

We tested the algorithm on 10 synthetic images, each one obtained with a mirror with a different profile. We used 10 lines in each image for calibration. In the worst case, the estimated $\alpha$ value was $1^{\circ}$ from the ground truth

The method has been used for calibrating an off axis catadioptric camera composed by a $3 \$$, off-the-shelf light bulb which has a reflective coating, and a 10Mpixel Canon EOS 400D camera. Figure 6 shows the experimental setting.

After calibrating the intrinsic camera parameters, we captured the image in Figure 7. The mirror apparent contour $\gamma$ and the four lines (Figure 7) used for minimization have been extracted using the Canny edge detector. Moreover, the harmonic homology has been computed from $\gamma$

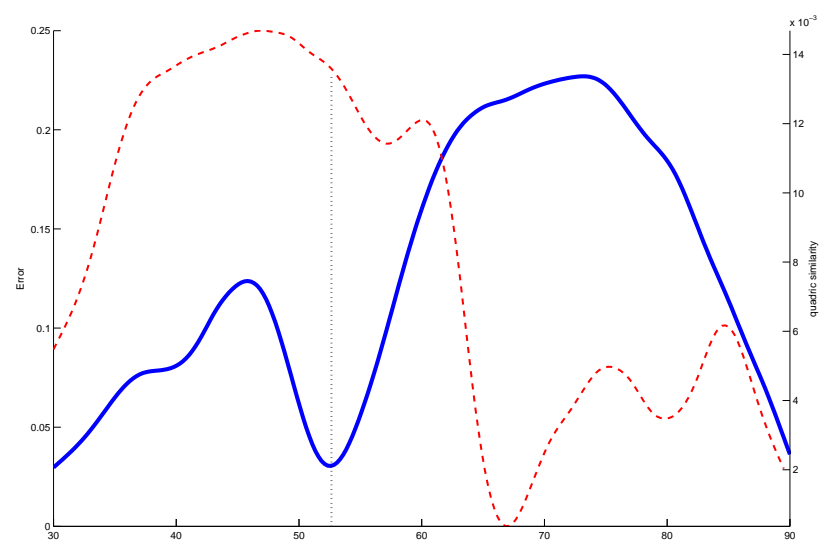

Figure 3. Solid line: error among a wide range of values for $\alpha$; dashed line: related quadric similarity of the viewing surface. Ground truth solution is shown as a vertical dashed line. Note that the reconstruction was only possible in a subset of the total $\alpha$ range $\left(-90^{\circ}, 90^{\circ}\right)$.

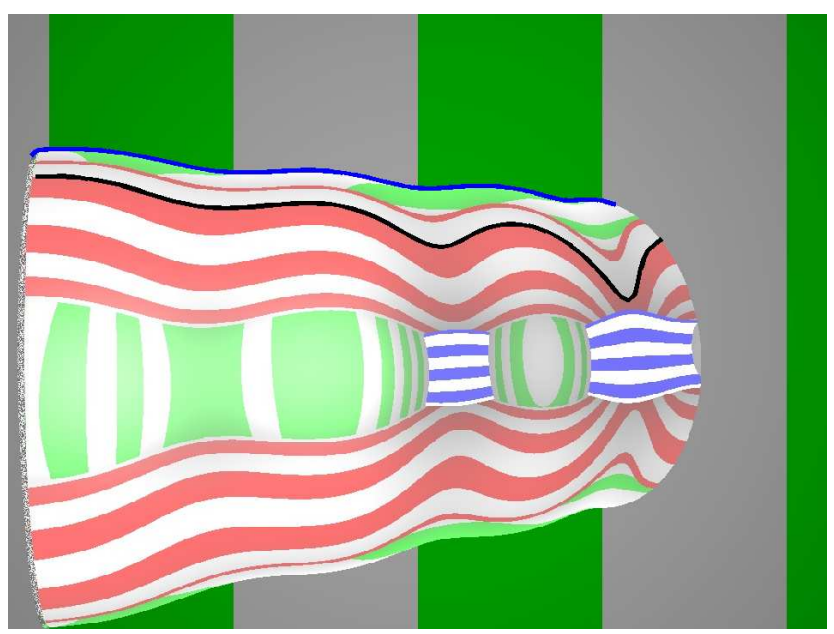

Figure 4. A second synthetic image showing the mirror, the extracted profile, represented in blue, and the used line image, shown in black. Also in this case the image of the visible cross section was not exploited

using [8], thus recovering $l$ and $v$. We explored the angle space from $10^{\circ}$ to $90^{\circ}$ at $1 / 6^{\circ}$ steps. The ground truth in camera test images has been measured (see Figure 6) as about $30^{\circ}$. The error term is minimized for $\alpha=31.67^{\circ}$ (see Figure 8), yielding satisfactory results.

\section{Conclusion and future works}

We presented a single-image calibration method for offaxis catadioptric cameras that only exploits the mirror apparent contour and at least one image of a generic space line. The algorithm has been successfully tested both on synthetic and camera images, confirming its correctness.

Further experiments are underway to explore the parameter space in the case of an uncalibrated natural perspective 


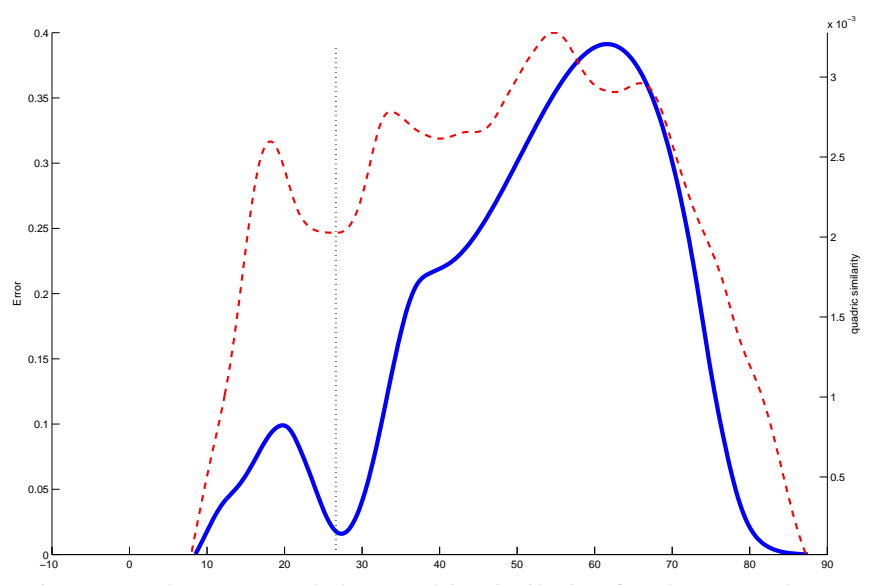

Figure 5. The error and the quadric similarity for the second experiment

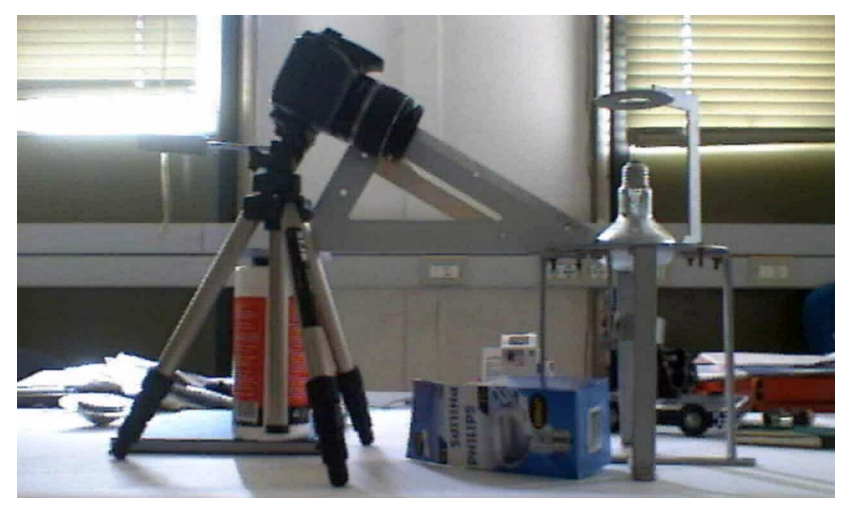

Figure 6. The lightbulb experimental setting

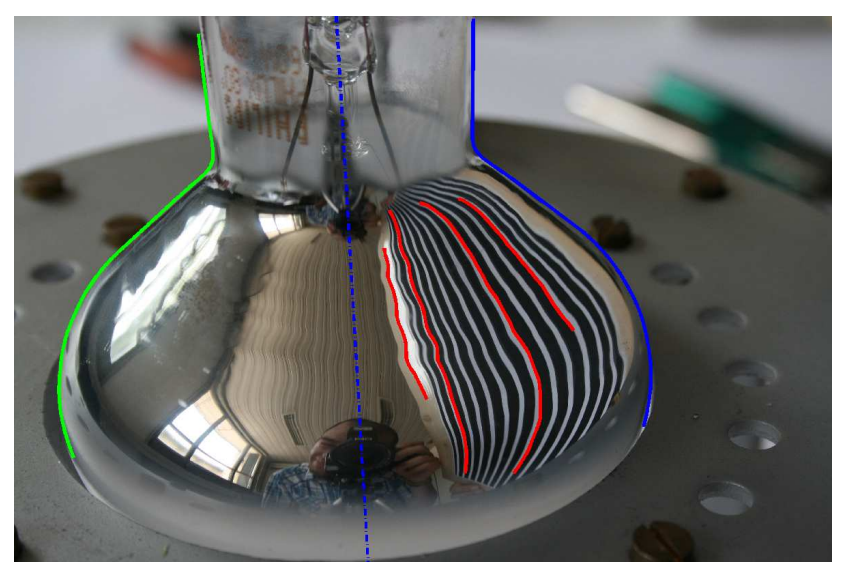

Figure 7. Source image for the lightbulb experiment. Red curves represent the four line images used for calibration.

camera. In this case, minimizing $e(k, \alpha)$ allows us to simultaneously calibrate all the unknown parameters.

Another possible extension is to consider a two mirror catadioptric system. In fact, whenever a space line is visible through both mirrors, we may exploit both viewing surfaces, which gives a better estimation of the reconstructed

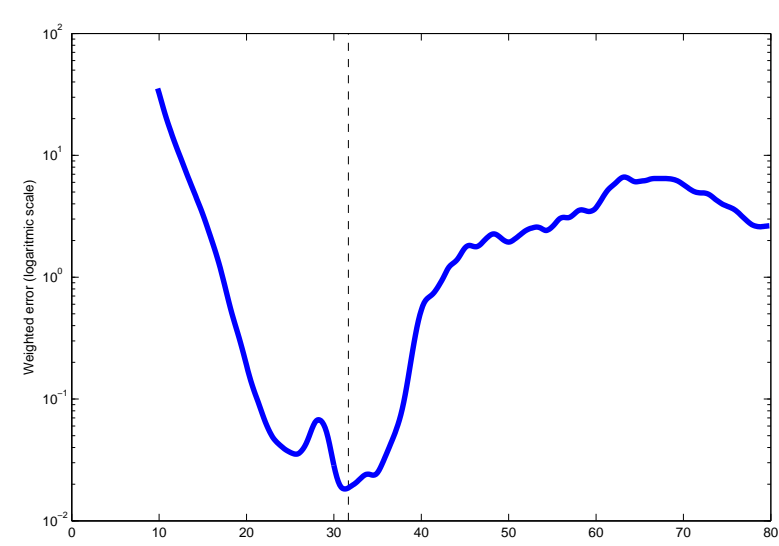

Figure 8 . The weighted mean error (in logarithmic scale). The function is minimized at $\alpha=31.67^{\circ}$

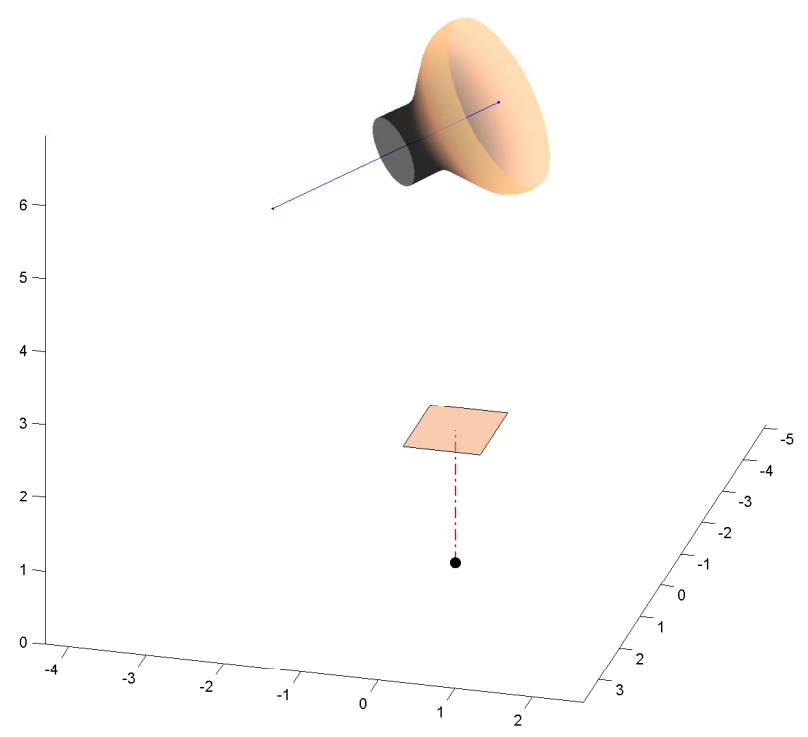

Figure 9. The calibration result showing the reconstructed mirror surface and its relative position w.r.t. the camera. The recovered angle is $\alpha=31.67^{\circ}$.

line [18] and a better estimation of the residual error for calibration.

\section{Acknowledgements}

The authors would thank Carlo Colombo and Dario Comanducci for providing us with the Matlab code to compute the harmonic homology of a solid of revolution. 


\section{References}

[1] D. Aliaga. Accurate catadioptric calibration for realtime pose estimation in room-size environments. In Proceedings of the IEEE International Conference on Computer Vision (ICCV 'O1), volume 1, pages 127134, Los Alamitos, CA, USA, 7-14 July 2001. IEEE Computer Society.

[2] S. Baker and S. K. Nayar. A theory of singleviewpoint catadioptric image formation. International Journal of Computer Vision, 35(2):175-196, November 1999.

[3] V. Caglioti and S. Gasparini. "How many planar viewing surfaces are there in noncentral catadioptric cameras?" Towards singe-image localization of space lines. In Proceedings of the IEEE International Conference on Computer Vision and Pattern Recognition (CVPR '06), volume 1, pages 1266-1273, Los Alamitos, CA, USA, 17-22 June 2006. IEEE Computer Society.

[4] V. Caglioti and S. Gasparini. Determining radius and position of a sphere from a single catadioptric image. To appear in: Journal of Intelligent and Robotic Systems: Theory and Applications, 2007.

[5] V. Caglioti, S. Gasparini, and P. Taddei. Methods for space line localization from single catadioptric images: new proposals and comparisons. In Proceedings of the 7th Workshop on Omnidirectional Vision (OMNIVIS 2007), 2007.

[6] J. Canny. A computational approach to edge detection. IEEE Transactions on Pattern Analysis and Machine Intelligence, 8(6):679-698, November 1986.

[7] Q. Chen, H. Wu, and T. Wada. Camera calibration with two arbitrary coplanar circles. In Proceedings of the European Conference on Computer Vision (ECCV '04), volume 3023/2004 of LCNS, pages 521-532. Springer, 2004.

[8] C. Colombo, A. D. Bimbo, and F. Pernici. Metric $3 \mathrm{~d}$ reconstruction and texture acquisition of surfaces of revolution from a single uncalibrated view. IEEE Transactions on Pattern Analysis and Machine Intelligence, 27(1):99-114, 2005.

[9] J. Fabrizio, J.-P. Tarel, and R. Benosman. Calibration of panoramic catadioptric sensors made easier. In Proceedings of the 3rd Workshop on Omnidirectional Vision (OMNIVIS 2002), pages 45-52, Los Alamitos, CA, USA, 2 June 2002. IEEE Computer Society.

[10] C. Geyer and K. Daniilidis. Paracatadioptric camera calibration. IEEE Transactions on Pattern Analysis and Machine Intelligence, 24(5):687-695, May 2002.
[11] D. Hilbert and S. Cohn-Vossen. Geometry and the Imagination. Chelsea Publishing Co., New York: Chelsea, 1932.

[12] K. Kanatani. Geometric computation for machine vision. Oxford University Press, Inc., New York, NY, USA, 1993.

[13] S. B. Kang. Catadioptric self-calibration. In Proceedings of the IEEE International Conference on Computer Vision and Pattern Recognition (CVPR 'O0), volume 1, pages 201-207, Los Alamitos, CA, USA, 1315 June 2000. IEEE Computer Society.

[14] D. Lanman, M. Wachs, G. Taubin, and F. Cukierman. Reconstructing a $3 \mathrm{~d}$ line from a single catadioptric image. In Proceedings of the Third International Symposium on 3D Data Processing, Visualization, and Transmission, pages 89-96, June 2006.

[15] T. Mashita, Y. Iwai, and M. Yachida. Calibration method for misaligned catadioptric camera. In Proceedings of the 6th Workshop on Omnidirectional Vision (OMNIVIS 2005), 2005. available online at http://www. eecs.berkeley . edu/ cgeyer/OMNIVIS05/.

[16] POV Team. Persistency of vision ray tracer (POVRay), http://www. povray.org.

[17] F. Sun, C. Yang, and Z. Hu. Planar conic based camera calibration. In Proceedings of the International Conference on Pattern Recognition (ICPR 'O0), page 1555, Washington, DC, USA, 2000. IEEE Computer Society.

[18] S. Teller and M. Hohmeyer. Determining the lines through four lines. Journal of graphics tools, 4(3):1122, 1999.

[19] K. Yamazawa, Y. Yagi, and M. Yachida. Omnidirectional imaging with hyperboloidal projection. In Proceedings of the IEEE/RSJ International Conference on Intelligent Robots and Systems, volume 2, pages 1029-1034, Los Alamitos, CA, USA, 26-30 July 1993. IEEE Computer Society.

[20] X. Ying and H. Zha. Linear catadioptric camera calibration from sphere images. In Proceedings of the 6th Workshop on Omnidirectional Vision (OMNIVIS 2005), 2005. available online at http://www. eecs . berkeley . edu/ cgeyer/OMNIVIS05/.

[21] X. Ying and H. Zha. Simultaneously calibrating catadioptric camera and detecting line features using hough transform. In Proceedings of the IEEE/RSJ International Conference on Intelligent Robots and Systems, pages 412-417, Los Alamitos, CA, USA, 2-6 Aug. 2005. IEEE Computer Society. 